\title{
Near-Real-Time Assessment of Cardiovascular Disease Risk Factors in Nebraska by Using ESSENCE
}

\author{
Sandra Gonzalez*1,2, Gary White ${ }^{1,2}$ and Tom Safranek ${ }^{1}$ \\ ${ }^{1}$ Nebraska Department of Health and Human Services, Lincoln, NE, USA; ${ }^{2}$ University of Nebraska-Lincoln, Lincoln, NE, USA
}

\section{Objective}

The main objective of this project is to expand inpatient (IP) syndromic surveillance in Nebraska to include indicators of Cardiovascular Disease (CVD).

\section{Introduction}

CVD is one of the leading causes of death in the US, with 800,000 deaths being linked to CVD every year. Recently, the CDC reported that 1 in 4 of these deaths could be prevented by lifestyle changes, creating healthier living spaces, as well as managing high cholesterol, blood pressure and diabetes levels [1]. The report also stressed the importance of electronic health records (EHR) in identifying patients with CVD risk factors [1].

Surveillance is a critical component of national effort to prevent CVD [2]. The Nebraska Department of Health and Human Services (NDHHS) has traditionally tracked the burden of chronic diseases by retrospective analysis of hospital discharge data (HDD). However, HDD is limited by its lack of immediate availability and its limited amount of data. Timeliness of detection and analysis of CVD events could be improved with syndromic surveillance. To enhance CVD surveillance in Nebraska, NDHHS implemented a near-real-time IP surveillance system in 2011. This surveillance system facilitates nearreal-time assessment of CVD risk factors, outcomes, and prevention program efficacy.

\section{Methods}

Previously the NDHHS described surveillance of CVD from IP data by using SAS statistical software to scan free text from key words present in chief complaints, triage notes and diagnostic codes [3]. Now we describe the detection, tracking and analysis of CVD events from near-real-time IP data by using Nebraska's ESSENCE system. An IP database was created in ESSENCE and algorithms were defined for the detection of myocardial infarction (MI) (defined by the ICD9 code 410), and for 5 risk factors for CVD: dyslipidemia, hypertension, diabetes, smoking, and obesity. The near-real-time EHR data were transmitted from one pilot hospital, Hospital A, which represents $10 \%$ of all IP admissions in Nebraska. The number of monthly MIs was tracked and the frequency of each risk factor within the MI cases analyzed. The quality of the data was assessed by calculating the percentage of completeness of the relevant data elements.

\section{Results}

A mean of 29 MIs per month (range 24-38) was detected in the IP data of Hospital A. The mean observed percentages for the CVD risks factors in these MI cases were 89\% (range 84-92), 86\% (range 70-97), $34 \%$ (range 19-59), 31\% (range 11-50), and 12\% (range 0-28) for hypertension, dyslipidemia, obesity, diabetes, and smoking, respectively. Data quality assessment demonstrated $100 \%$ of completeness for required data elements and a relatively high percent of completeness for most data elements relevant to dyslipidemia, hypertension, diabetes, and obesity (100\% for primary ICD9 diagnostic code, $95 \%$ for height and weight, $75 \%$ for active medication list and $75 \%$ for smoking status).

\section{Conclusions}

ESSENCE allows the monitoring of risk factors and CVD events, in a fraction of time needed for data processing and analysis by using SAS statistical software. Results of this study demonstrate the applicability of ESSENCE for near-real-time monitoring of risk factors and CVD events. Such timely detection holds potential to enhance prospective monitoring of CVD trends in populations and impact efforts towards CVD prevention. The results suggest a need to focus efforts on the prevention of obesity and improvement in the management of dyslipidemia and hypertension. However, this study represents only $10 \%$ of the IP admissions in Nebraska, and might not be representative of the state population. Overall, this study suggests that the use of ESSENCE for monitoring IP data can improve the accuracy and timeliness of the detection, tracking, and analysis of CVD events and improve the identification of at-risk populations and the development of effective prevention plans.

Hospital A

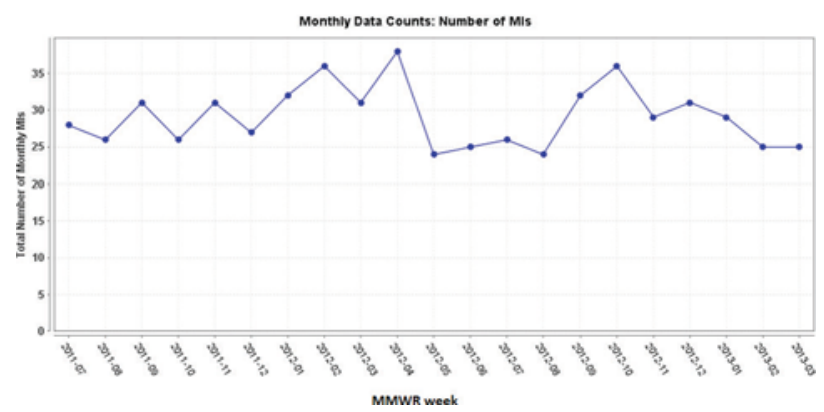

Keywords

Syndromic Surveillance; Cardiovascular Disease; ESSENCE

\section{References}

[1] CDC, Vital signs, "Preventable Deaths from Heart Disease \& Stroke: Improving Care Can Save More Lives," www.cdc.gov/VitalSigns/pdf/2013-09-vitalsigns.pdf, accessed September 5, 2013

[2] CDC MMWR December 21, 2012. "CDC Grand Rounds: The Million Hearts Initiative," www.cdc.gov/mmwr/PDF/wk/mm6150.pdf, accessed September 5, 2013

[3] Close N and Murphy E (2012) Meaningful Use Webinar Series "Discovering the New Frontier of Syndromic Surveillance: A Meaningful Use Dialogue on the Nebraska and Washington Implementations", ISDS, http://vimeo.com/38180890, accessed September 5, 2013

\footnotetext{
*Sandra Gonzalez

E-mail: Sandra.Gonzalez@nebraska.gov
} 